\title{
CHEMICAL ABUNDANCES OF THE LEO II DWARF GALAXY
}

\author{
Matthew D. Shetrone ${ }^{1}$, Michael H. Siegel ${ }^{2}$, David O. CooK $^{3}$, and Tammy Bosler ${ }^{4,5}$ \\ ${ }^{1}$ University of Texas, McDonald Observatory, HC75 Box 1337-McD, Fort Davis, TX 79734, USA \\ ${ }^{2}$ University of Texas, McDonald Observatory, Austin, TX 78712, USA \\ ${ }^{3}$ Department of Astronomy, University of Minnesota, 116 Church Street SE, Minneapolis, MN 55455, USA \\ ${ }^{4}$ Division of Astronomical Sciences, National Science Foundation, VA, USA \\ Received 2008 April 10; accepted 2008 October 2; published 2008 December 2
}

\begin{abstract}
We use previously published moderate-resolution spectra in combination with stellar atmosphere models to derive the first measured chemical abundance ratios in the Leo II dwarf Spheroidal (dSph) galaxy. We find that for spectra with signal-to-noise ratio greater than 24 , we are able to measure abundances from weak $\mathrm{Ti}$, Fe, and $\mathrm{Mg}$ lines located near the calcium infrared triplet (CaT). We also quantify and discuss discrepancies between the metallicities measured from $\mathrm{Fe}$ I lines and those estimated from the CaT features. We find that while the most metal-poor $([\mathrm{Fe} / \mathrm{H}]<-2.0])$ Leo II stars have $\mathrm{Ca}$ and $\mathrm{Ti}$ abundance ratios similar to those of Galactic globular clusters, the more metal-rich stars show a gradual decline of $\mathrm{Ti}, \mathrm{Mg}$, and $\mathrm{Ca}$ abundance ratio with increasing metallicity. Finding these trends in this distant and apparently dynamically stable dSph galaxy supports the hypothesis that the slow chemical enrichment histories of the $\mathrm{dSph}$ galaxies is universal, independent of any interaction with the Milky Way. Combining our spectroscopic abundances with published broadband photometry and updated isochrones, we are able to approximate stellar ages for our bright red giant branch stars to a relative precision of 2-3 Gyr. While the derived age-metallicity relationship of Leo II hints at some amount of slow enrichment, the data are still statistically consistent with no enrichment over the history of Leo II.
\end{abstract}

Key words: galaxies: dwarf - galaxies: evolution - galaxies: individual (Leo II) - stars: abundances

Online-only material: color figures

\section{INTRODUCTION}

Dwarf galaxies are the most common type of galaxy in the universe and are thought to be the progenitors of larger structures. Therefore, a clear understanding of dwarf galaxy evolution is the first step toward a larger understanding of general galaxy evolution. Fortunately, dwarf galaxies are also comparatively simple systems that can be effectively modeled and simulated. In particular, the satellite galaxies of the Milky Way, by virtue of being nearby, compact, and situated in the sparser regions of the Galactic halo, have proven to be exceptional laboratories for studying models of stellar and chemical evolution (see e.g., Mateo 1998).

A useful tool for studying the dwarf galaxies is the comparison of their chemical properties to those of the wellstudied Milky Way globular clusters. Some globular clusters show evidence of multiple stellar populations (Omega Centauri, Lee et al. 1999; NGC 1851, Milone et al. 2008; NGC 2808, Piotto et al. 2007). Others, particularly those associated with dwarf Spheroidal (dSph) galaxies or tidal streams, show peculiar chemical enrichment histories and/or young ages (see e.g., Letarte et al. 2006). However, most inner halo Milky Way globular clusters formed their stars a Hubble time ago in a single burst (Marin-Franch et al. 2008). Since the early universe was dominated by massive stars, it is likely that only core-collapse supernovae (type II SN) were able to enrich the primordial interstellar medium (ISM) before the globular clusters formed. These events would have enriched the ISM with both Fe and $\alpha$-elements, resulting in relatively high $\alpha$-element to iron ratios $[\alpha / \mathrm{Fe}]$. By contrast, objects that formed later, after type Ia SN had time to enrich the ISM with more Fe, would have lower relative $\alpha$-element ratios.

\footnotetext{
5 AAAS Science \& Technology Policy Fellow.
}

Most dwarf galaxies have compound populations, having undergone slow sporadic star formation over their lifetimes (see review in Mateo 1998). While their earliest populations were formed in the aftermath of the first SNII events, their later populations benefited from ongoing SNIa. The result is a steep decline in $\alpha$-element abundance ratios with decreasing age/increasing metallicity. The most metal-poor stars have $\alpha$-element ratios similar to globular clusters, while the more metal-rich populations are deficient in $\alpha$-elements (Shetrone et al. 1998, 2001, 2003; Tolstoy et al. 2003; Venn et al. 2004; Smith et al. 2006; Helmi et al. 2006).

The chemistry of dSph stars is of particular importance in untangling the hierarchical formation of the Milky Way. Cold dark matter cosmological models predict that galaxies like the Milky Way have grown through the merging of low-mass systems with perhaps some low-level merging continuing at the present time. Among the current retinue of dSph satellite galaxies, the Sagittarius dSph shows clear signs of having been torn apart by the Milky Way potential (Majewski et al. 2003) and Ursa Minor, Leo I, Coma Berenices, and Ursa Major II also hint at a violent dynamical past (Palma et al. 2003; Sohn et al. 2007; Simon \& Geha 2007).

However, while the most metal-poor stars of the dSph galaxies are chemically similar to the most metal-poor stars of the Galactic halo, the enrichment of SNIa kicks in at lower Fe abundances in the $\mathrm{dSph}$ galaxies, resulting in consistent deficits in the $\alpha$-element ratios of dSph stars compared to halo stars of similar $[\mathrm{Fe} / \mathrm{H}]$ (Venn et al. 2004). This would indicate that the Galactic halo formed from objects that had more rapid and therefore more SNII-dominated chemical evolution and that objects like the current dSph galaxies have contributed little to the halo.

This argument is, however, undermined by recent studies indicating that the stars the Sagittarius dSph is contributing 
to the halo differ from those in the residual core (Bellazzini et al. 2006; Chou et al. 2007; Siegel et al. 2007). If the dSph galaxies are the remnants of much larger objects that have been disrupted over a Hubble time, the present stellar populations of the dSph galaxies may not reflect the initial stellar populations they would have preferentially contributed to the Galactic halo. Moreover, if the stellar populations of the dSph galaxies have not evolved in isolation, their chemical abundances may have been affected by continual dynamical harassment.

These arguments over dSph stellar chemistry could be clarified by examination of more distant dwarf galaxies that have not been perturbed by the Milky Way potential. If an isolated dSph were shown to have the same chemical abundance patterns as the relatively nearby dSphs, such a discovery would (1) strengthen the argument that the lack of halo stars with "dSph-like" abundance patterns suggests that the vast majority of the halo formed very quickly and that the dSphs have contributed very few stars since this initial construction phase and (2) show that the $\alpha$-element deficits have nothing to do with dynamical stirring and are entirely the product of dSph stellar and chemical evolution.

In an effort to address these issues, we now expand the study of chemical abundances to the Leo II dSph galaxy. Leo II is the second most distant dSph galaxy assumed to be orbiting the Milky Way (218 kpc, Bellazzini et al. 2005; Siegel et al. 2008, hereafter S08). It is predominantly metal-poor (Hodge 1982; Demers \& Harris 1983; Aaronson et al. 1983; Azzopardi et al. 1985; Suntzeff et al. 1986; Lee 1995; Demers \& Irwin 1993; Koch et al. 2007a, 2007b; Bosler et al. 2007, hereafter BSS07) and dominated by intermediate age populations (Mighell \& Rich 1996; Dolphin et al. 2005; Bellazzini et al. 2005; Gullieuszik et al. 2008). It has a handful of carbon stars (Azzopardi et al. 1985), a rich population of RR Lyrae variable stars (Swope 1967, 1968; Siegel \& Majewski 2000), a high central velocity dispersion (Vogt et al. 1995; Koch et al. 2007b; BSS07; S08), and an extended stellar distribution (Coleman et al. 2007; S08). At present, however, the indications are that it had undergone little, if any, interaction with the Galactic potential (Koch et al. 2007b; Coleman et al. 2007; Walker et al. 2007; S08) and its modest radial velocity $\left(+26.2 \mathrm{~km} \mathrm{~s}^{-1}, \mathrm{~S} 08\right)$ would be consistent with a circular orbit that does not bring it close to the Milky Way.

The BSS07 study cited above analyzed medium-resolution NIR spectra of a large number of Leo II red giant branch (RGB) stars in an effort to measure radial velocities and metallicities. These spectra are dominated by the Calcium II infrared triplet (CaT) features but also have numerous weak $\mathrm{Fe}, \mathrm{Ti}$, and $\mathrm{Mg}$ lines that have yet to be exploited. In this paper, we show that these weak lines can be used to measure metallicity and abundance ratios for stars of sufficient signal-to-noise ratio $(\mathrm{S} / \mathrm{N})$ through the use of synthetic model spectra. This method has allowed us to study the chemical evolution of Leo II, to constrain the relationship between the equivalent width of the $\mathrm{CaT}$ lines and the $[\mathrm{Fe} / \mathrm{H}]$ abundances as measured from Fe I lines and to make a preliminary examination of the agemetallicity relationship (AMR) of the brightest Leo II RGB stars.

Section 2 discusses the additional reduction and analysis we have applied to the BSS07 data. Section 3 compares the abundances ratios measured in Leo II to those measured in the globular clusters to provide insight into the chemical evolution of Leo II. Section 4 examines the inferred ages of our bright Leo II stars, while Section 5 summarizes our findings.

\section{SPECTROSCOPIC REDUCTION AND ANALYSIS TECHNIQUES}

A full description of the observations and data reduction can be found in BSS07 but we give a brief summary of the observations here. Low-dispersion spectra of red giants in the Leo II dSph were taken using the Keck I $10 \mathrm{~m}$ telescope and LRIS (Oke et al. 1995) in 2002 and 2003. The LRIS was configured with the $12001 \mathrm{~mm}^{-1}$ grating blazed at $7500 \AA$ which gives a dispersion of $0.62 \AA$ per pixel and a resolution of $1.55 \AA(R \sim 10,000)$. Twenty to thirty stars were observed on each slit mask creating a total sample of 74 stars with $\mathrm{S} / \mathrm{N}$ between 10 and 44 .

Traditional equivalent width analysis of these spectra proved impossible due to the heavy blending of many lines and the difficulty in setting the continuum measure. However, these problems can be overcome by comparison of the observed spectra to synthetic spectra created by the 2007 version of the LTE line-analysis code MOOG (Sneden 1973).

The initial line list was obtained from the R. L. Kurucz CD-ROM 23. In order to further calibrate this line list we compared the list with the nearby red giant star, Arcturus (Hinkle et al. 2000). We adopted atmospheric parameters for Arcturus $\left(T_{\text {eff }}=4280 \mathrm{~K}, \log g=1.55, v_{t}=1.61 \mathrm{~km} \mathrm{~s}^{-1},[\mathrm{Fe} / \mathrm{H}]=-0.50\right.$, $[\mathrm{Ti} / \mathrm{Fe}]=+0.26,[\mathrm{Mg} / \mathrm{Fe}]=+0.39$, and $[\mathrm{Ca} / \mathrm{Fe}]=+0.21)$ by combining the results of McWilliam (1990), Fulbright et al. (2006, 2007), and Koch \& McWilliam (2008). Since we are performing a differential analysis, we chose to adjust the $g f$ values in our synthetic spectrum only for lines that were significantly discrepant from the catalog Arcturus spectrum, rather than adjust all 8345 lines.

We smoothed the model spectra with a Gaussian kernel of FWHM equivalent to the BSS07 spectral resolution of $1.55 \AA$. Initial metallicity estimates for globular cluster and Leo II stars were taken from BSS07. Effective temperatures were calculated from interpolation of the temperature-colormetallicity relationship of Ramírez \& Meléndez (2005) and the $B V$ photometry of S08, the latter allowing the Leo II and cluster data to be analyzed using the same photometric system.

Surface gravities were calculated from the temperatures and bolometric-corrected magnitudes assuming a mass of $0.8 M_{\odot}$. We used distance moduli of $15.07,13.76,15.59$, 15.19, and 21.61 for M3, M107, NGC 1094, NGC 4590, and Leo II, respectively. ${ }^{6}$ The microturbulence was estimated as $v_{t}=-0.41 \times \log g+2.15$, in accordance with recent analyses of globular cluster giants near the tip of the giant branch (e.g., Sneden et al. 2004; Lee et al. 2005; Ivans et al. 2001; Cohen $\&$ Meléndez 2005). Microturbulent velocities ranged from 1.4 to $2.0 \mathrm{~km} \mathrm{~s}^{-1}$ in the globular cluster sample and from 1.6 to $2.0 \mathrm{~km} \mathrm{~s}^{-1}$ in the Leo II sample.

These parameters were fed into MOOG to produce preliminary synthetic spectra. We then adjusted the $[\mathrm{Fe} / \mathrm{H}]$ metallicity of the atmospheres until the Fe lines matched the observed spectrum. A weighted mean of all the iron lines was used to determine the best $[\mathrm{Fe} / \mathrm{H}]$ value for each star.

The model was then set to this metallicity and the abundance ratios were determined by holding all elements constant while varying the desired $\alpha$-element. The best fit was determined by the output difference between the MOOG synthetic spectra and observed spectra. We found that we were able to effectively

\footnotetext{
6 We find in Section 4 that Leo II's distance modulus is likely closer to 21.7. Correcting the Leo II distance modulus would change the inferred $\log g$ values by approximately 0.04 , an insignificant correction.
} 


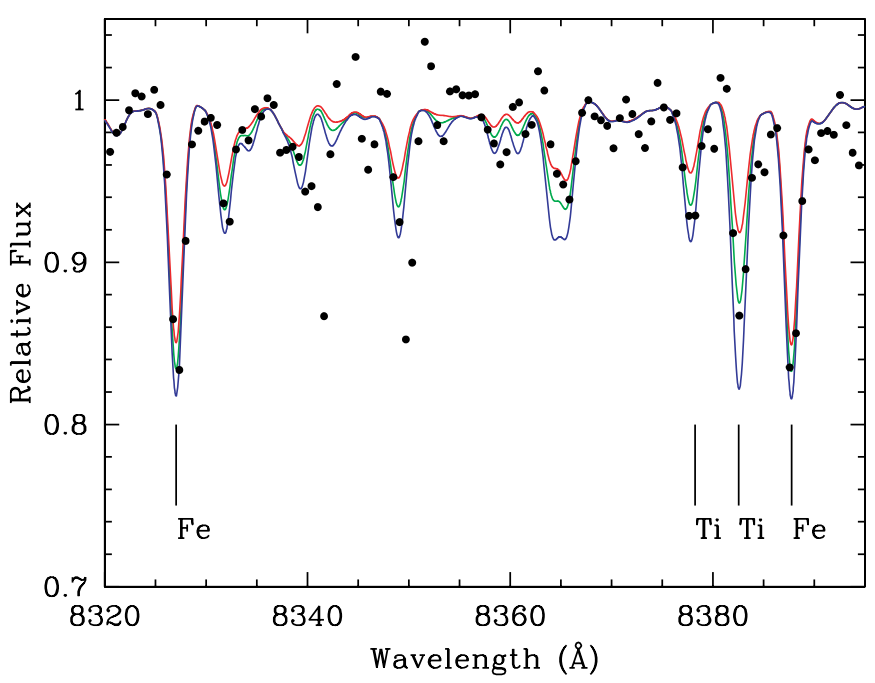

Figure 1. Modeling the stellar spectra with MOOG. The points represent the spectrum of Leo II star 180 , one of the higher $\mathrm{S} / \mathrm{N}$ spectra. The lines represent synthetic spectra with 0.3 dex higher (blue) and lower (red) Fe and $\mathrm{Ti}$ abundances. Two Ti lines and two Fe lines used in this analysis are labeled.

(A color version of this figure is available in the online journal.)

measure abundances for stars with $\mathrm{S} / \mathrm{N}$ greater than 24 per pixel.

The setting of the continuum was critical for a proper analysis of the weak lines in these medium-resolution spectra. To refine the continuum levels we created a synthetic spectrum for each star, divided it into the observed spectrum and fit the residual with a high-order spline. This fitted spline was then divided into the observed spectrum to remove high-order terms in the continuum placement. If our modeled spectrum changed significantly, e.g., when a new overall metallicity change was required, we repeated this procedure for the new model.

We attempted to determine abundance ratios for all of the moderate lines in the spectra. These lines include Fe, Ca, Na, $\mathrm{Mg}$, and Ti. Figure 1 compares one of our highest $\mathrm{S} / \mathrm{N}$ spectra to several synthetic spectra with varied $\mathrm{Fe}$ and $\mathrm{Ti}$ abundances. There are a large number of lines in this region with varying strength that can be used to determine abundances. For a more typical S/N spectrum, most of these lines are not strong enough for abundance analysis. We identified sufficient $\mathrm{Ti}$ and $\mathrm{Fe}$ lines in all of our bright stars but the $\mathrm{Na}$ lines and all but one of the $\mathrm{Mg}$ lines were absent in most of the spectra. Thus we limit our analysis to the stronger $\mathrm{Fe}, \mathrm{Ca}, \mathrm{Mg}$, and $\mathrm{Ti}$ lines listed in Table 1.

The only $\mathrm{Ca}$ lines in the observed spectral region are the extremely strong $\mathrm{CaT}$ lines. The CaT lines are difficult to model with synthetic spectra (hence the more common use of globular cluster-calibrated equivalent width analysis). At a constant $\mathrm{Ca}$ abundance, the strength of the CaT lines decreases as the electron pressure increases due to changes in the continuous opacities. For cool stellar atmospheres, the main sources of electrons are $\mathrm{Mg}, \mathrm{Fe}, \mathrm{Si}, \mathrm{Ca}, \mathrm{Na}$, and $\mathrm{Al}$, but which element contributes the most depends in the effective temperature of the star and which layer of the atmosphere is surveyed. For example, for a $T_{\text {eff }}=4200 \mathrm{~K}$ star at 0.01 optical depth, the main sources of electrons are $\mathrm{Mg}$ and $\mathrm{Fe}$; while for a $T_{\text {eff }}=4000 \mathrm{~K}$ star at 0.01 optical depth, the main sources of electrons are $\mathrm{Ca}, \mathrm{Mg}, \mathrm{Na}$, and Al. Thus, as the $\alpha$-abundance declines, the continuous opacity changes to counteract the $\mathrm{Ca}$ abundance decline. For example, a 0.5 dex change in the electron contributors will increase the $\mathrm{CaT}$ line strength enough to mimic a $\mathrm{Ca}$ abundance increase
Table 1

Main Lines Used in Abundance Analysis

\begin{tabular}{lccr}
\hline \hline Element & Wavelength & $\mathrm{eV}$ & $\log g f$ \\
\hline Ti I & 8378.25 & 3.72 & -2.30 \\
Ti I & 8382.53 & 0.82 & -1.63 \\
Ti I & 8435.65 & 0.84 & -1.30 \\
Fe I & 8220.38 & 4.32 & 0.20 \\
Fe I & 8327.06 & 2.20 & -1.64 \\
Fe I & 8387.77 & 2.18 & -1.60 \\
Fe I & 8468.41 & 2.22 & -2.17 \\
Fe I & 8514.07 & 2.20 & -2.25 \\
Fe I & 2.85 & -2.15 \\
Fe I & 8611.80 & 2.83 & -2.00 \\
Fe I & 2.18 & -1.21 \\
Mg I & 8674.75 & 4.35 & -0.13 \\
Ca II & 8688.63 & 1.69 & -1.31 \\
Ca II & 8806.76 & 1.70 & -0.36 \\
Ca II & 8498.02 & 1.69 & -0.62 \\
\hline
\end{tabular}

of 0.38 and 0.22 dex for stars of $T_{\text {eff }}=4000 \mathrm{~K}, \log g=0.7$, $[\mathrm{M} / \mathrm{H}]=-1.1$, and $T_{\text {eff }}=4200 \mathrm{~K}, \log g=0.7,[\mathrm{M} / \mathrm{H}]=-1.9$, respectively. This means that as $[\mathrm{M} / \mathrm{H}]$ rises, any decline in the $[\mathrm{Ca} / \mathrm{Fe}]$ ratio would be muted in the measured $\mathrm{CaT}$ lines. As a further complication, the CaT lines become less sensitive to the $\mathrm{Ca}$ abundance as the lines strengthen.

For the globular cluster sample, the Plez model atmospheres with $[\alpha / \mathrm{Fe}]=+0.4$ enhanced abundance ratios were appropriate and there was no need to change the model. However, our initial analysis showed that the $\mathrm{Ti}$ and $\mathrm{Mg}$ ratios of the Leo II stars decline with increasing metallicity (Section 3). This resulted in stars with low $\alpha$-abundances having erroneously high $\mathrm{Ca}$ abundances. To derive accurate $\mathrm{Ca}$ abundance, we were therefore required to iterate. We first determined the $\mathrm{Fe}$ abundance for each star and then adjusted the model appropriately. We next determined the Ti and Mg abundances, from which we calculated a preliminary $\alpha$-abundance. Finally, we adjusted the model abundance based on the average $\alpha$-abundance at each star's $[\mathrm{Fe} / \mathrm{H}]$. The result was that for the more metal-rich Leo II stars we used model atmospheres that were more metal-poor than the Fe abundance would imply, thus compensating for the lack of electrons owing to the lower $\alpha$-abundances.

Measurement errors were determined for each fitted feature by adjusting the abundances up and down until the residuals of the fit were larger than the surrounding continuum regions, i.e., larger than the $\mathrm{S} / \mathrm{N}$. The measurement errors were then propagated to yield a single measurement error which was added in quadrature to the abundance errors from the modeling uncertainties- $\pm 100 \mathrm{~K}$ for $T_{\text {eff }}, \pm 0.2$ dex for $\log g, \pm 0.25$ for $v_{t}$, and \pm 0.2 dex for $[\mathrm{M} / \mathrm{H}]$. Typically, the measurement errors dominated over the modeling errors particularly for $[\mathrm{Mg} / \mathrm{Fe}]$ which was derived from a single spectral feature.

Tables 2 and 3 list the globular cluster and Leo II stars from BSS07 for which we have been able to determine abundances, while Tables 4 and 5 show the abundances derived from the MOOG software. We have recalculated the $\mathrm{S} / \mathrm{N}$ from the residual of the best-fit synthetic spectra to the observed spectra and list that $\mathrm{S} / \mathrm{N}$ in Tables 2 and 3.

\section{SPECTROSCOPIC RESULTS}

Figure 2 shows the photometrically derived effective temperatures and surface gravities for the Leo II sample. There is a clear RGB sequence, corresponding to the photometric 
Table 2

Cluster Sample Taken From the Bosler Survey

\begin{tabular}{|c|c|c|c|c|c|c|}
\hline Stars & $V(\mathrm{mag})$ & $B-V$ & $\mathrm{~S} / \mathrm{N}$ & $T_{\text {eff }}(\mathrm{K})$ & $\log g$ & $v_{t}$ \\
\hline \multicolumn{7}{|c|}{ NGC 1904} \\
\hline 241 & 13.61 & 1.11 & 37.6 & 4419 & 0.94 & 1.77 \\
\hline 131 & 13.02 & 1.20 & 34.9 & 4298 & 0.85 & 1.80 \\
\hline 223 & 13.19 & 1.16 & 34.5 & 4350 & 0.72 & 1.85 \\
\hline 160 & 14.06 & 1.52 & 31.2 & 3969 & 0.31 & 2.02 \\
\hline 294 & 14.22 & 1.00 & 22.3 & 4586 & 1.29 & 1.62 \\
\hline 181 & 13.95 & 1.25 & 21.3 & 4236 & 0.94 & 1.76 \\
\hline \multicolumn{7}{|c|}{ NGC 4590} \\
\hline HI82 & 12.59 & 1.33 & 24.6 & 4188 & 0.34 & 1.98 \\
\hline HI119 & 13.62 & 0.96 & 21.1 & 4633 & 1.09 & 1.70 \\
\hline HI239 & 14.19 & 0.87 & 14.2 & 4788 & 0.89 & 1.79 \\
\hline \multicolumn{7}{|c|}{ M3 } \\
\hline 265 & 13.26 & 1.30 & 93.0 & 4155 & 0.83 & 1.81 \\
\hline 250 & 14.11 & 0.95 & 82.1 & 4627 & 1.50 & 1.54 \\
\hline 640 & 13.27 & 1.26 & 77.1 & 4199 & 0.87 & 1.79 \\
\hline 589 & 12.87 & 1.33 & 76.6 & 4124 & 0.64 & 1.89 \\
\hline 1217 & 14.00 & 1.05 & 76.4 & 4470 & 1.36 & 1.59 \\
\hline 885 & 13.47 & 1.07 & 75.5 & 4441 & 1.13 & 1.69 \\
\hline 334 & 13.24 & 1.20 & 55.3 & 4269 & 0.91 & 1.78 \\
\hline 238 & 12.69 & 1.57 & 53.5 & 3918 & 0.35 & 2.01 \\
\hline \multicolumn{7}{|c|}{ M107 } \\
\hline Sl & 14.04 & 1.47 & 56.1 & 4581 & 1.37 & 1.59 \\
\hline Sf & 13.39 & 1.70 & 49.0 & 4225 & 0.87 & 1.79 \\
\hline $\mathrm{Sr}$ & 14.66 & 1.29 & 42.2 & 4910 & 1.80 & 1.41 \\
\hline Sh & 13.84 & 1.61 & 38.9 & 4356 & 1.15 & 1.68 \\
\hline Ss & 14.79 & 1.40 & 37.9 & 4703 & 1.74 & 1.43 \\
\hline $\mathrm{Su}$ & 14.78 & 1.28 & 37.5 & 4930 & 1.86 & 1.39 \\
\hline S62 & 13.97 & 1.62 & 34.1 & 4341 & 1.19 & 1.66 \\
\hline
\end{tabular}

Table 3

Leo II Sample Taken From the Bosler Survey

\begin{tabular}{|c|c|c|c|c|c|c|c|}
\hline Stars & $V$ (mag) & $B-V$ & $\mathrm{~S} / \mathrm{N}$ & {$[\mathrm{M} / \mathrm{H}]_{\mathrm{CaT}}{ }^{\mathrm{a}}$} & $T_{\text {eff }}(\mathrm{K})$ & $\log g$ & $v_{t}$ \\
\hline 180 & 18.998 & 1.5117 & 43.7 & -1.60 & 3981 & 0.33 & 2.02 \\
\hline 255 & 19.291 & 1.3114 & 40.0 & -1.66 & 4151 & 0.62 & 1.90 \\
\hline 271 & 19.375 & 1.3561 & 39.5 & -1.48 & 4100 & 0.60 & 1.90 \\
\hline 258 & 19.311 & 1.3507 & 36.9 & -1.57 & 4110 & 0.59 & 1.91 \\
\hline 336 & 19.531 & 1.1669 & 36.8 & -2.03 & 4299 & 0.83 & 1.81 \\
\hline 195 & 19.026 & 1.0915 & 36.2 & -1.60 & 4406 & 0.71 & 1.86 \\
\hline 296 & 19.385 & 1.0488 & 35.7 & -2.02 & 4438 & 0.87 & 1.79 \\
\hline 293 & 19.304 & 1.2481 & 35.5 & -1.84 & 4216 & 0.68 & 1.87 \\
\hline 166 & 18.827 & 1.1629 & 35.3 & -1.52 & 4318 & 0.56 & 1.92 \\
\hline 285 & 19.337 & 1.3657 & 35.0 & -1.50 & 4091 & 0.58 & 1.91 \\
\hline 304 & 19.389 & 1.2711 & 34.7 & -1.81 & 4194 & 0.69 & 1.87 \\
\hline 254 & 19.259 & 1.3981 & 33.6 & -1.43 & 4055 & 0.51 & 1.87 \\
\hline 236 & 19.212 & 1.3330 & 32.6 & -1.90 & 4144 & 0.58 & 1.91 \\
\hline 351 & 19.552 & 1.2835 & 32.5 & -1.40 & 4176 & 0.74 & 1.85 \\
\hline 333 & 19.495 & 1.2278 & 32.4 & -1.45 & 4240 & 0.77 & 1.83 \\
\hline 379 & 19.618 & 1.3171 & 32.1 & -1.52 & 4141 & 0.74 & 1.85 \\
\hline 209 & 19.033 & 1.4749 & 31.7 & -1.59 & 4006 & 0.37 & 2.00 \\
\hline 256 & 19.332 & 1.2784 & 30.4 & -1.54 & 4182 & 0.66 & 1.88 \\
\hline 341 & 19.541 & 1.2780 & 29.2 & -1.43 & 4182 & 0.74 & 1.85 \\
\hline 281 & 19.342 & 1.1127 & 28.6 & -2.05 & 4358 & 0.80 & 1.82 \\
\hline 282 & 19.329 & 1.4611 & 27.6 & -1.52 & 4008 & 0.49 & 1.95 \\
\hline 420 & 19.717 & 1.0824 & 27.1 & -1.46 & 4430 & 1.00 & 1.74 \\
\hline 377 & 19.587 & 1.1853 & 25.8 & -1.73 & 4283 & 0.84 & 1.80 \\
\hline 260 & 19.268 & 1.2811 & 25.7 & -1.57 & 4179 & 0.63 & 1.89 \\
\hline 248 & 19.354 & 1.2399 & 25.1 & -1.51 & 4225 & 0.70 & 1.86 \\
\hline 234 & 19.250 & 1.2238 & 24.5 & -1.80 & 4240 & 0.67 & 1.87 \\
\hline 230 & 19.194 & 1.4458 & 24.1 & -1.56 & 4025 & 0.45 & 1.96 \\
\hline
\end{tabular}

Note. ${ }^{a}$ Based on the BSS07 CaT measurement and the Carretta \& Gratton (1997) metallicity scale.
Table 4

Derived Abundances for Cluster Sample

\begin{tabular}{|c|c|c|c|c|}
\hline Star & {$[\mathrm{Fe} \mathrm{I} / \mathrm{H}](\sigma)$} & {$[\mathrm{Ti} \mathrm{I} / \mathrm{Fe} \mathrm{I}](\sigma)$} & {$[\mathrm{Mg} \mathrm{I} / \mathrm{Fe} \mathrm{I}](\sigma)$} & {$[\mathrm{Ca}$ II $/ \mathrm{Fe} \mathrm{I}](\sigma)$} \\
\hline \multicolumn{5}{|c|}{ NGC 1904} \\
\hline 241 & $-1.70(0.18)$ & $0.11(0.27)$ & $0.30(0.38)$ & $0.70(0.17)$ \\
\hline 131 & $-1.89(0.21)$ & $0.70(0.25)$ & $0.45(0.37)$ & $0.70(0.20)$ \\
\hline 223 & $-1.55(0.18)$ & $0.57(0.22)$ & $0.50(0.39)$ & $0.70(0.17)$ \\
\hline 160 & $-2.06(0.26)$ & $-0.03(0.30)$ & $0.70(0.59)$ & $0.35(0.20)$ \\
\hline 294 & $-1.62(0.22)$ & $0.65(0.27)$ & $0.60(0.42)$ & $0.45(0.17)$ \\
\hline 181 & $-2.02(0.22)$ & $0.46(0.25)$ & $0.20(0.43)$ & $0.85(0.24)$ \\
\hline Average & $-1.77(0.08)$ & $0.44(0.10)$ & $0.44(0.17)$ & $0.61(0.08)$ \\
\hline \multicolumn{5}{|c|}{ NGC 4590} \\
\hline HI82 & $-2.44(0.16)$ & $0.13(0.20)$ & $0.50(0.30)$ & $0.50(0.22)$ \\
\hline HI119 & $-2.25(0.14)$ & $0.22(0.20)$ & $0.70(0.24)$ & $0.35(0.27)$ \\
\hline HI239 & $-2.03(0.18)$ & $0.28(0.37)$ & $-0.20(0.35)$ & $0.30(0.22)$ \\
\hline Average & $-2.26(0.09)$ & $0.19(0.13)$ & $0.44(0.17)$ & $0.39(0.13)$ \\
\hline \multicolumn{5}{|c|}{ M3 } \\
\hline 265 & $-1.62(0.14)$ & $-0.08(0.16)$ & $0.20(0.25)$ & $0.60(0.17)$ \\
\hline 238 & $-2.10(0.19)$ & $0.30(0.18)$ & $0.10(0.32)$ & $0.45(0.19)$ \\
\hline 250 & $-1.40(0.14)$ & $0.38(0.16)$ & $0.50(0.28)$ & $0.40(0.15)$ \\
\hline 334 & $-1.71(0.14)$ & $0.36(0.16)$ & $0.30(0.22)$ & $0.75(0.15)$ \\
\hline 589 & $-1.77(0.19)$ & $0.43(0.16)$ & $0.30(0.27)$ & $0.65(0.15)$ \\
\hline 640 & $-1.51(0.14)$ & $-0.02(0.16)$ & $0.30(0.28)$ & $0.55(0.15)$ \\
\hline 885 & $-1.61(0.15)$ & $0.40(0.17)$ & $0.05(0.34)$ & $0.45(0.16)$ \\
\hline 1217 & $-1.68(0.16)$ & $0.60(0.20)$ & $0.10(0.23)$ & $0.50(0.18)$ \\
\hline Average & $-1.64(0.05)$ & $0.28(0.06)$ & $0.24(0.09)$ & $0.55(0.06)$ \\
\hline \multicolumn{5}{|c|}{ M107 } \\
\hline S62 & $-1.03(0.19)$ & $0.55(0.23)$ & $0.50(0.27)$ & $0.50(0.20)$ \\
\hline $\mathrm{Sf}$ & $-0.94(0.18)$ & $0.60(0.23)$ & $0.15(0.26)$ & $0.40(0.20)$ \\
\hline $\mathrm{Sh}$ & $-1.18(0.16)$ & $0.72(0.20)$ & $0.35(0.24)$ & $0.50(0.17)$ \\
\hline $\mathrm{Sl}$ & $-1.17(0.21)$ & $0.68(0.26)$ & $0.35(0.33)$ & $0.50(0.22)$ \\
\hline $\mathrm{Sr}$ & $-1.01(0.20)$ & $0.55(0.24)$ & $0.25(0.27)$ & $0.35(0.21)$ \\
\hline Ss & $-1.05(0.19)$ & $0.89(0.27)$ & $0.10(0.29)$ & $0.40(0.20)$ \\
\hline$\underline{\mathrm{Su}}$ & $-0.92(0.23)$ & $0.13(0.40)$ & $-0.10(0.33)$ & $0.15(0.24)$ \\
\hline Average & $-1.05(0.07)$ & $0.63(0.09)$ & $0.25(0.11)$ & $0.41(0.08)$ \\
\hline
\end{tabular}

Table 5

Derived Abundances for Leo II Sample

\begin{tabular}{|c|c|c|c|c|}
\hline Star & {$[\mathrm{Fe} \mathrm{I} / \mathrm{H}](\sigma)$} & {$[\mathrm{Ti}$ I/Fe I] $(\sigma)$} & {$[\mathrm{Mg} \mathrm{I} / \mathrm{Fe} \mathrm{I}](\sigma)$} & {$[\mathrm{Ca} \mathrm{II} / \mathrm{Fe} \mathrm{I}](\sigma)$} \\
\hline 180 & $-1.94(0.18)$ & $-0.16(0.16)$ & . & $0.25(0.15)$ \\
\hline 209 & $-1.97(0.17)$ & $-0.13(0.16)$ & $-0.20(0.30)$ & $0.30(0.15)$ \\
\hline 271 & $-1.77(0.18)$ & $-0.10(0.18)$ & $0.20(0.30)$ & $0.50(0.17)$ \\
\hline 351 & $-1.73(0.22)$ & $-0.17(0.21)$ & $0.25(0.28)$ & $0.30(0.18)$ \\
\hline 166 & $-1.85(0.18)$ & $0.18(0.19)$ & $0.10(0.30)$ & $0.60(0.16)$ \\
\hline 293 & $-1.93(0.18)$ & $-0.05(0.17)$ & $-0.20(0.15)$ & $0.35(0.15)$ \\
\hline 336 & $-1.98(0.21)$ & $\ldots$ & $0.10(0.32)$ & $0.20(0.17)$ \\
\hline 236 & $-2.26(0.22)$ & $0.33(0.21)$ & $0.50(0.33)$ & $0.40(0.20)$ \\
\hline 195 & $-1.70(0.19)$ & $0.36(0.23)$ & $0.70(0.30)$ & $0.42(0.18)$ \\
\hline 258 & $-1.64(0.19)$ & $-0.17(0.19)$ & $0.05(0.30)$ & $0.30(0.17)$ \\
\hline 254 & $-1.80(0.19)$ & $-0.10(0.18)$ & $0.40(0.27)$ & $0.20(0.17)$ \\
\hline 255 & $-2.05(0.19)$ & $0.30(0.17)$ & $-0.35(0.39)$ & $0.45(0.16)$ \\
\hline 296 & $-2.13(0.19)$ & $0.28(0.22)$ & $0.30(0.33)$ & $0.40(0.17)$ \\
\hline 304 & $-2.09(0.18)$ & $-0.02(0.18)$ & $0.45(0.27)$ & $0.35(0.16)$ \\
\hline 281 & $-2.07(0.21)$ & $0.35(0.26)$ & $0.15(0.36)$ & $0.35(0.20)$ \\
\hline 282 & $-1.68(0.19)$ & $-0.34(0.19)$ & $-0.30(0.37)$ & $0.05(0.17)$ \\
\hline 333 & $-1.95(0.21)$ & $0.28(0.21)$ & $0.35(0.30)$ & $0.50(0.19)$ \\
\hline 285 & $-1.73(0.18)$ & $-0.03(0.20)$ & $0.05(0.28)$ & $0.45(0.16)$ \\
\hline 379 & $-1.71(0.21)$ & $-0.03(0.21)$ & $0.15(0.33)$ & $0.30(0.20)$ \\
\hline 260 & $-1.75(0.21)$ & $-0.17(0.20)$ & $\ldots$ & $0.35(0.18)$ \\
\hline 341 & $-1.42(0.19)$ & $-0.18(0.19)$ & $-0.00(0.30)$ & $0.15(0.17)$ \\
\hline 256 & $-1.73(0.18)$ & $0.05(0.18)$ & $-0.20(0.16)$ & $0.35(0.16)$ \\
\hline 248 & $-1.72(0.20)$ & $0.03(0.21)$ & $0.05(0.18)$ & $0.40(0.19)$ \\
\hline 420 & $-1.80(0.22)$ & $0.24(0.25)$ & $0.45(0.32)$ & $0.45(0.21)$ \\
\hline 230 & $-1.88(0.24)$ & $0.07(0.25)$ & $0.75(0.34)$ & $0.50(0.23)$ \\
\hline 377 & $-1.98(0.20)$ & $0.09(0.24)$ & $0.15(0.38)$ & $0.40(0.19)$ \\
\hline 234 & $-1.76(0.19)$ & $-0.20(0.21)$ & $-0.10(0.33)$ & $0.25(0.18)$ \\
\hline
\end{tabular}




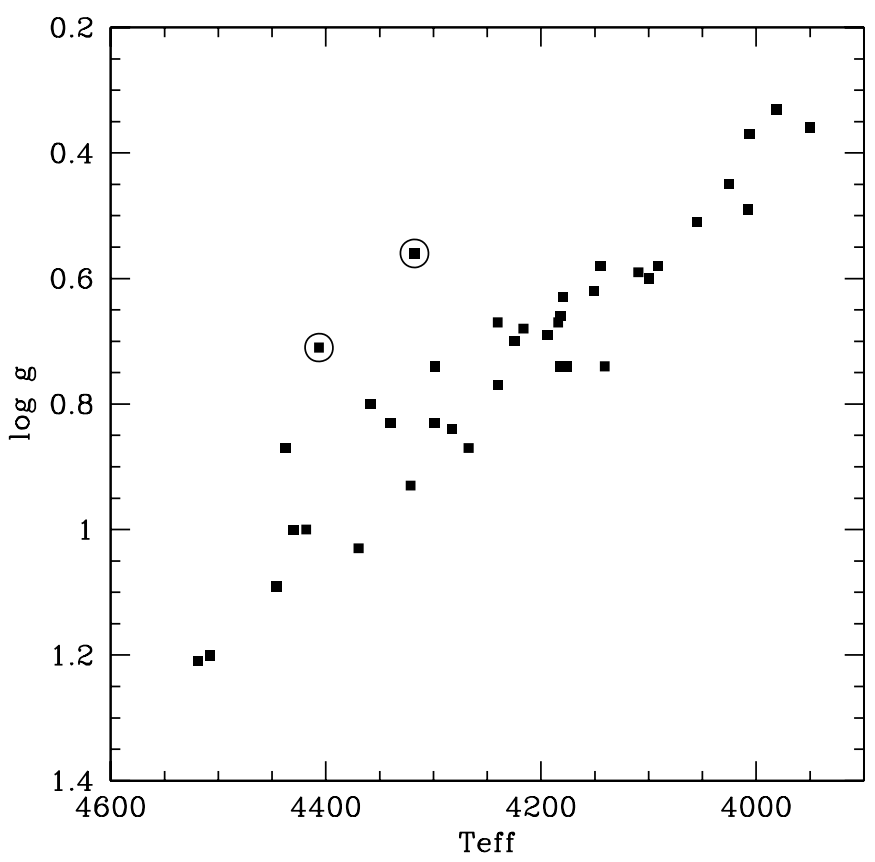

Figure 2. Hertzsprung-Russell diagram of the Leo II stars. The two stars with large circles are stars 166 and 195 which are radial velocity members but do not seem to fall on the RGB locus and thus are suspect for our analysis.

sequence upon which the temperatures and gravities are based. The two outlier stars-195 and 166 - are metal-poor radial velocity members. They are, however, according to S08, well removed from the dominant Leo II RGB locus in color-magnitude space although both have Washington+DDO51 photometry consistent with giant stars.

It is possible that these are interloping giants in the field, possibly from the metal-poor debris stream of the Sagittarius $\mathrm{dSph}$ galaxy passing in front of Leo II (S08). Alternatively, they could represent a young, metal-poor population in Leo II (Section 4). Given their outlier status, we have chosen to remove these two stars from the analysis of mean trends in the Leo II sample.

Figure 3 compares our derived $[\mathrm{Fe} / \mathrm{H}]$ metallicities against those derived from the CaT lines by BSS07. On average, the CaT-based metallicities are 0.17 dex higher than our model $[\mathrm{Fe} / \mathrm{H}]$ metallicities with an rms of $0.18 \mathrm{dex}$. The abundances are correlated but there is a positive residual which shows increasing significance with decreasing metallicity.

The offset is likely due to several factors. First, all of our $\mathrm{Fe}$ lines are $\mathrm{Fe} \mathrm{I}$ lines and it is possible that metal-poor giants overionize Fe (Thévenin \& Idiart 1999; Asplund \& García Pérez 2001). In M3, for example, Sneden et al. (2004) find an underabundance factor of -0.13 in the Fe I lines and they adopt the Fe II abundances as the true metallicity. In M68, Lee et al. (2005) find an underabundance factor of -0.37 in the Fe I lines and also adopt the Fe II abundances as the true metallicity. However, not all authors find these over-ionization factors. Cohen \& Meléndez (2005) find that the Fe I and Fe II abundances agree to within uncertainties. Kraft \& Ivans (2003) find small and variable overionization in metal-poor globular clusters but of much smaller magnitude than Thévenin \& Idiart (1999) predict. In light of the disagreement, we have chosen not to make any correction for over-ionization but will discuss the potential impact in later sections.

A second potential source of the discrepancy between the $[\mathrm{M} / \mathrm{H}]_{\mathrm{CaT}}$ and our derived $[\mathrm{Fe} / \mathrm{H}]$ comes from the calibration

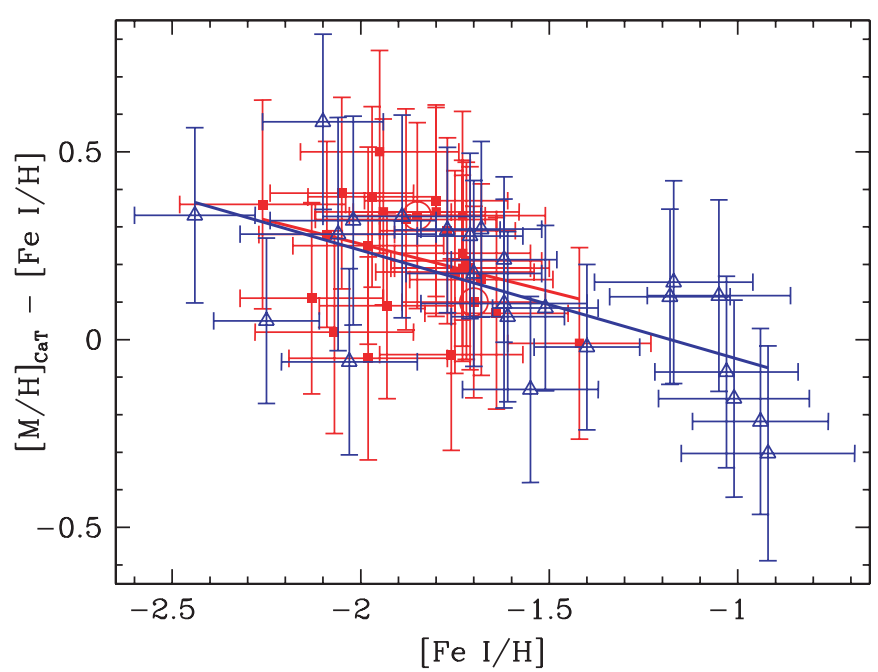

Figure 3. $[\mathrm{Fe} / \mathrm{H}]$ computed from this analysis are compared against the CaT metallicities for the Leo II stars (red filled squares) and the globular clusters (blue open triangles). The thick lines are the best-fit lines to the data. The two stars with large circles are stars 166 and 195.

(A color version of this figure is available in the online journal.)

of the CaT metallicity scale, which in BSS07 is based on the Carretta \& Gratton (1997) metallicity scale. The choice of calibration system can significantly impact the zero point of the metallicity. Battaglia et al. (2008), for example, find a 0.1 dex difference between the Carretta \& Gratton (1997) metallicity scale and their high-resolution analysis over the range of metallicity spanned by Leo II's RGB stars.

We can explore the issue of the metallicity zero point by comparing the Fe I metallicities we derive for the BSS07 globular clusters to the high-resolution analyses listed in Pritzl et al. (2005). The average abundances we derive for NGC 1904, NGC 4590, and M3 are $-0.14(\sigma=0.22)$ dex more metalpoor than those listed in Pritzl et al. However, their NGC 1904 abundances come solely from the study of Gratton \& Ortolani (1989). Pritzl et al. find that the Gratton \& Ortolani abundances are typically 0.16 more metal-rich than more recent analyses. Correcting the NGC 1904 abundance down by 0.16 dex, we find our calculated Fe I abundances for NGC 1904, NGC 4590, and M3 are $-0.08(0.14)$ dex more metal-poor than the Pritzl et al. values, a zero-point offset within the error of the weighted mean abundances listed in Table 4.

Figures 4 and 5 show the derived $[\mathrm{Ti} / \mathrm{Fe}]$ and $[\mathrm{Mg} / \mathrm{Fe}]$ abundance ratios, respectively, for both the Leo II and globular cluster sample. The Leo II and globular cluster star [Ti/Fe] abundances overlap at the poorest metallicities but depart dramatically at higher metallicities. The Leo II $[\mathrm{Mg} / \mathrm{Fe}]$ ratios are offset from the globular cluster sample and show a pronounced decline with increasing metallicity. The large error bars on the $[\mathrm{Mg} / \mathrm{Fe}]$ limit the usefulness of the individual measurements but confirm the same trend seen in the $[\mathrm{Ti} / \mathrm{Fe}]$ abundance ratios.

We note that the $[\mathrm{Ti} / \mathrm{Fe}]$ ratios in $\mathrm{M} 107$ are as high if not higher than those found in the more metal-poor globular clusters. Such large $[\mathrm{Ti} / \mathrm{Fe}]$ abundance ratios are not unprecedented. Sneden et al. (1994) found that the relatively metal-rich globular cluster M71 had high $\mathrm{Ti}$ abundance ratios $([\mathrm{Ti} / \mathrm{Fe}]=+0.5)$, although Ramírez \& Cohen (2002) found "normal" Ti ratios M71.

Figure 6 shows the $[\mathrm{Ca}$ II $/ \mathrm{Fe}$ I] abundance ratios for the globular cluster and Leo II samples. There is a slight decline in the $[\mathrm{Ca}$ II $/ \mathrm{Fe} \mathrm{I}]$ ratio with increasing metallicity among the 


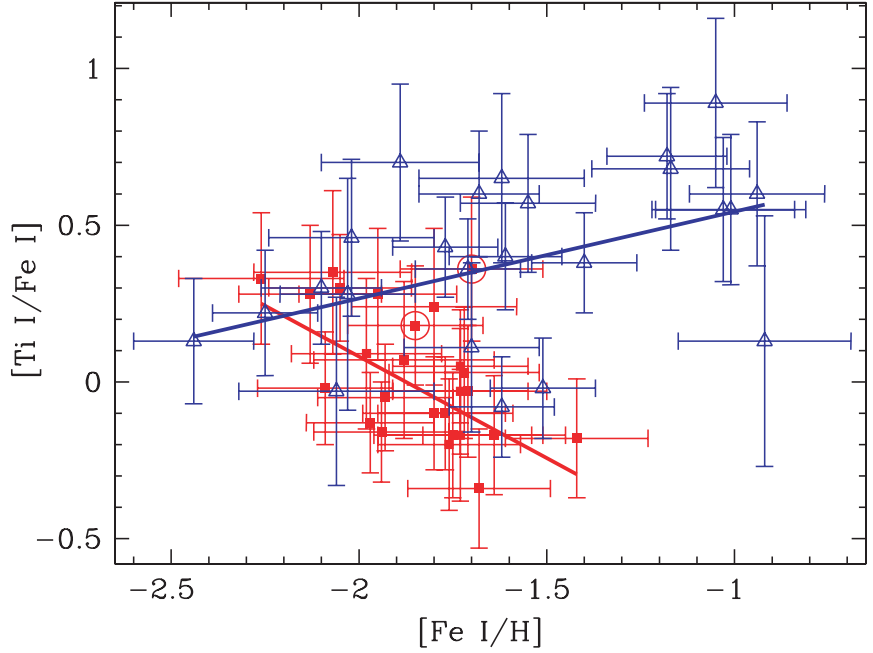

Figure 4. $[\mathrm{Ti} / \mathrm{Fe}]$ computed from this analysis is shown for the Leo II stars (red filled squares) and the globular clusters (blue open triangles). The thick lines are the best-fit lines to the data. The two stars with large circles are stars 166 and 195 .

(A color version of this figure is available in the online journal.)

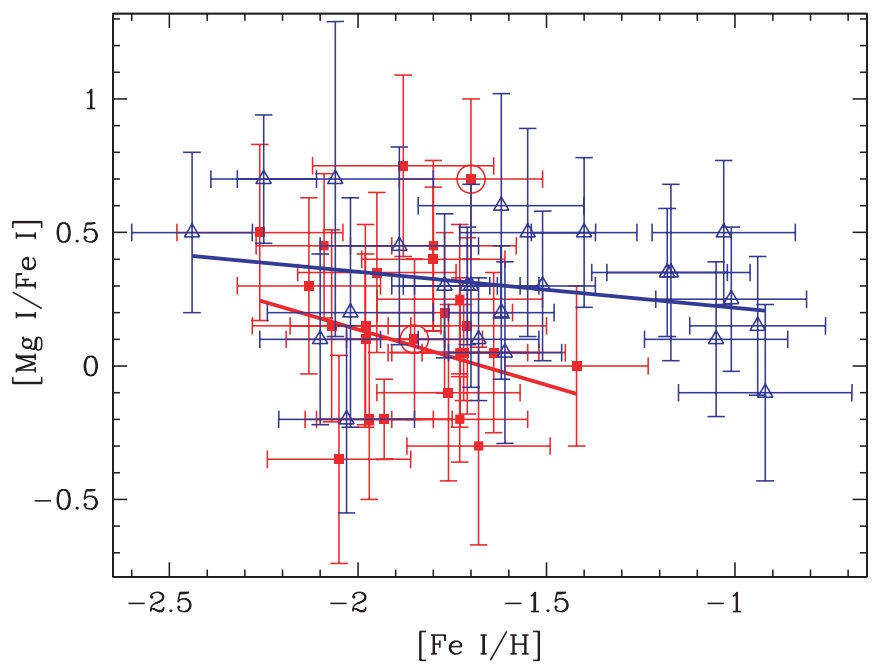

Figure 5. $[\mathrm{Mg} / \mathrm{Fe}]$ computed from this analysis is shown for the Leo II stars (red filled squares) and the globular clusters (blue open triangles). The thick lines are the best-fit lines to the data. The two stars with large circles are stars 166 and 195.

(A color version of this figure is available in the online journal.)

globular cluster sample. The Leo II Ca abundance ratios are offset from the globular cluster sample and the slope of the best-fit line is slightly steeper than that of the cluster sample. This is very similar to the trends in $\mathrm{Mg}$ abundances seen in Figure 5.

For M3, NGC 4590, and NGC 1904, we compare our abundance ratios to the compilation of Pritzl et al. (2005). Our $[\mathrm{Ti} / \mathrm{Fe}]$ ratios are $0.09 \pm 0.05$ dex larger, our $[\mathrm{Mg} / \mathrm{Fe}]$ ratios are $0.01 \pm 0.05$ dex larger, and our $[\mathrm{Ca} / \mathrm{Fe}]$ ratios are $0.24 \pm 0.13$ dex larger. The only significant zero point is in our $[\mathrm{Ca} \mathrm{II} / \mathrm{Fe} \mathrm{I}]$ abundances. This is not entirely unexpected. Most analyses compare ionized species to ionized species and neutral species to neutral species. We would obviously prefer to make a similar comparison, but the previously noted lack of Fe II lines prevents this. Additionally, as noted above, the literature is divided on the over-ionization correction need to converted from $\mathrm{Fe}$ I to $\mathrm{Fe}$ II abundances. Such corrections are

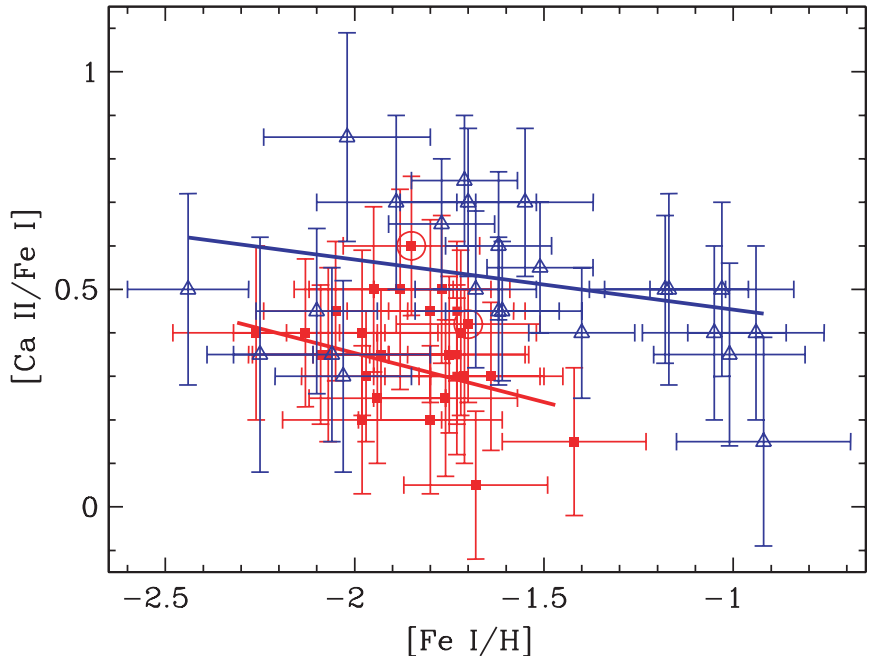

Figure 6. [Ca II/Fe I] computed from this analysis is shown for the Leo II stars (red filled squares) and the globular clusters (blue open triangles). The thick lines are the best-fit lines to the data. The two stars with large circles are stars 166 and 195. Please see Section 3 and Figure 7 for a full discussion of the errors and zero points associated with the $\mathrm{Ca}$ abundances shown in this figure.

(A color version of this figure is available in the online journal.)

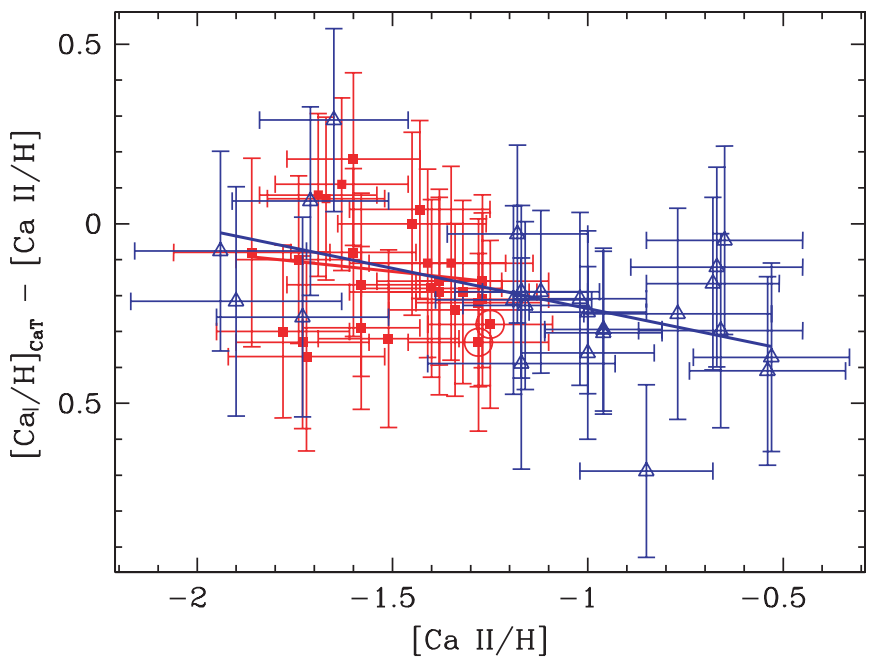

Figure 7. $[\mathrm{Ca} / \mathrm{H}]$ computed from this analysis are compared against the CaT $[\mathrm{Ca} / \mathrm{H}]$ metallicities for the Leo II stars (red filled squares) and the globular clusters (blue open triangles) from BSS07. The thick lines are the best-fit lines to the data. The two stars with large circles are stars 166 and 195.

(A color version of this figure is available in the online journal.)

likely to be metallicity dependent and could be the cause of the slope in the globular cluster sample. Because we do not make a correction, the absolute value of our $[\mathrm{Ca}$ II $/ \mathrm{Fe} \mathrm{I}]$ abundances is uncertain. However, because our analysis is concerned with relative differences between the clusters and Leo II, such a zeropoint correction will have little impact on our conclusions.

Figure 7 shows the $[\mathrm{Ca} / \mathrm{H}]$ ratios derived from our analysis compared to the $[\mathrm{Ca} / \mathrm{H}]$ ratios derived from the CaT by BSS07. The overlap of the cluster and Leo II sample in this figure suggests that the corrections we made for the more metal-rich low- $\alpha$ stars are consistent with $\mathrm{Ca}$ abundances derived with the $\mathrm{CaT}$ methodology. The slopes in these samples are less steep than those of Figure 3-as would be expected since the CaT lines should be sensitive to the overall $\mathrm{Ca}$ abundance. However, there is a residual slope between the two $[\mathrm{Ca} / \mathrm{H}]$ scales, which remains unexplained. 
Figure 8 shows the mean of the $[\mathrm{Ca} / \mathrm{Fe}],[\mathrm{Ti} / \mathrm{Fe}]$, and $[\mathrm{Mg} / \mathrm{Fe}]$ ratios. The fit to the data with the lowest formal reduced $\chi^{2}$ is a second-order Legendre polynomial which exhibits a steep decline in the $\alpha$-abundance ratios of the Leo II stars with metallicities above $[\mathrm{Fe} / \mathrm{H}] \sim-2.0$. This is consistent with what is seen in other dSph galaxies (see references in Section 1) and is likely due to slower chemical evolution and concordant increased contribution of SNIa to Leo II. However, the data would also be consistent with a step function near $[\mathrm{Fe} / \mathrm{H}] \sim-1.8$ in which the metal-poor stars have globular cluster like $\alpha$-abundances, while the more metal-rich stars are underabundant in $\alpha$ elements.

\section{AGE DISTRIBUTION OF LEO II STARS}

The slow enrichment implied by our abundance analysis suggests that Leo II should have an AMR in which the more metal-rich stars are younger than the more metal-poor stars and have therefore been subject to more enrichment by SNIa. An AMR would not be immediately obvious in colormagnitude diagrams due to the photometric degeneracy of age and metallicity. However, our precise spectroscopic survey affords the ability to remove abundance from the equation. We can now combine precise broadband photometry with modern isochrones to approximate individual stellar ages. This method has had some success in untangling the stellar populations of the Omega Centauri globular cluster, for example (Hilker et al. 2004; Sollima et al. 2005), and was recently applied to the Leo II dSph galaxy by Koch et al. (2007a).

The Koch et al. study found that Leo II has undergone steady star formation with little enrichment, starting $9 \mathrm{Gyr}$ ago and continuing up until $2 \mathrm{Gyr}$ ago. More recently formed stars appear to be somewhat enriched. Our analysis uses similar data but with the advantage that we have measured $\alpha$-abundances, rather than assumed abundances.

Because our stars are near the tip of the RGB (TRGB), rather than the more age-sensitive turnoff, any analysis will necessarily be imprecise (Koch et al. estimate uncertainties of $40 \%$ using these techniques). The ideal stars for this analysis are those near the age-sensitive main sequence turnoff (MSTO). However, given that Leo II's MSTO is well beyond the spectroscopic range of even the largest ground-based telescopes, the RGB is our best option for probing Leo II's age distribution.

Figure 9 demonstrates the analysis. Photometry is taken from S08, which has a precision of $0.01 \mathrm{mag}$ in all passbands at the TRGB. V I colors were calculated by converting the Washington measures in S08 using transformations in Majewski et al. (2000). Isochrones are taken from the Dartmouth Stellar Evolution Program (Dotter et al. 2007) and shifted to a distance modulus of $(m-M)=21.70$ and reddening of $E(B-V)=0.02$, in accordance with S08's TRGB distance derived from the same data.

Cursory examination of the individual panels, broken down by passband and metallicity bin, shows no obvious AMR in Leo II. To make a more precise estimate, we interpolated the isochrones in $0.01 \mathrm{mag}$ intervals and, with metallicity fixed at the spectroscopic value and $[\alpha / \mathrm{Fe}]$ fixed to the mean $[\alpha / \mathrm{Fe}]$ at each star's $[\mathrm{Fe} / \mathrm{H}]$, we found the isochrone nearest to each star's photometric position. We estimated age uncertainties by offsetting the $[\mathrm{Fe} / \mathrm{H}]$ of each star by its uncertainty (which dominates over S08's small photometric uncertainties).

The left panels of Figure 10 show the resultant age-metallicity distribution. Although the uncertainties are significant (typically

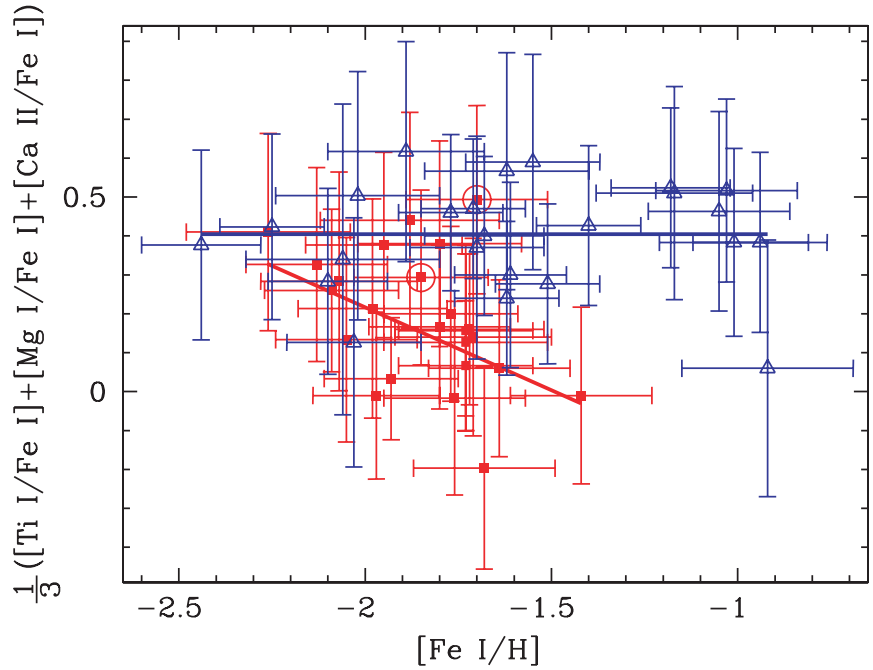

Figure 8. Average $\alpha$-abundance for the globular cluster (open blue triangles) and Leo II samples (red filled squares) are shown from the unweighted $\mathrm{Ca}, \mathrm{Mg}$, and $\mathrm{Ti}$ abundance ratios. The thick lines are the best-fit lines to the data. The two stars with large circles are stars 166 and 195 and are not included in the best fit.

(A color version of this figure is available in the online journal.)

2-3 Gyr), some interesting trends can be seen. The mean age of the stars is $\sim 9$ Gyr but has a spread significantly greater than the uncertainty, implying ongoing low-level star formation. The younger stars appear to be slightly more metal-rich and indeed, an AMR that shows slight enrichment with age-either gradually or step-wise-would be more consistent with the data than a flat trend. However, we cannot statistically rule out a flat AMR, which would have a $\chi^{2}$ per degree of freedom of 0.9 . In fact, our derived AMR is very similar to that of Koch et al. (2007a).

It should be noted that the exploration of the RGB stars has a number of complications that could affect the inferred AMR. In particular,

1. Leo II is known to have prominent asymptotic giant branch (AGB; Mighell \& Rich 1996; Gullieuszik et al. 2008). Stars on the AGB will overlap young RGB isochrones and perhaps $10 \%$ of our stars could be AGB stars. The most likely candidates would be the two apparently young metal-poor stars that are clear outliers in the bottom panels of Figure 9 and both panels of Figure 10 (note that these are different stars than the two outliers rejected in Figures 2-8, which were excluded a priori from the age analysis). We attempted to measure ages for these stars using the Padova isochrones (Marigo et al. 2008), which include an AGB. However, these stars are too blue for all but the youngest AGB isochrones (at these magnitudes, the RGB and AGB are separated by only a few $0.01 \mathrm{mag}$ ). Removing these outlier stars from the sample, however, increases the contrast between the younger and older stars of Leo II, making enrichment more tenable. But a flat AMR would still be within the uncertainties $\left(\chi^{2}=0.9\right)$.

2. Any age analysis will be sensitive to the distance modulus of Leo II. Adjusting the distance of Leo II shifts the absolute ages, but not the relative ages. The exception is at short distance moduli, where the stars are too faint to correspond to anything but the maximum age in our isochrone set (15 Gyr). If we assume that Leo II's stars must be 


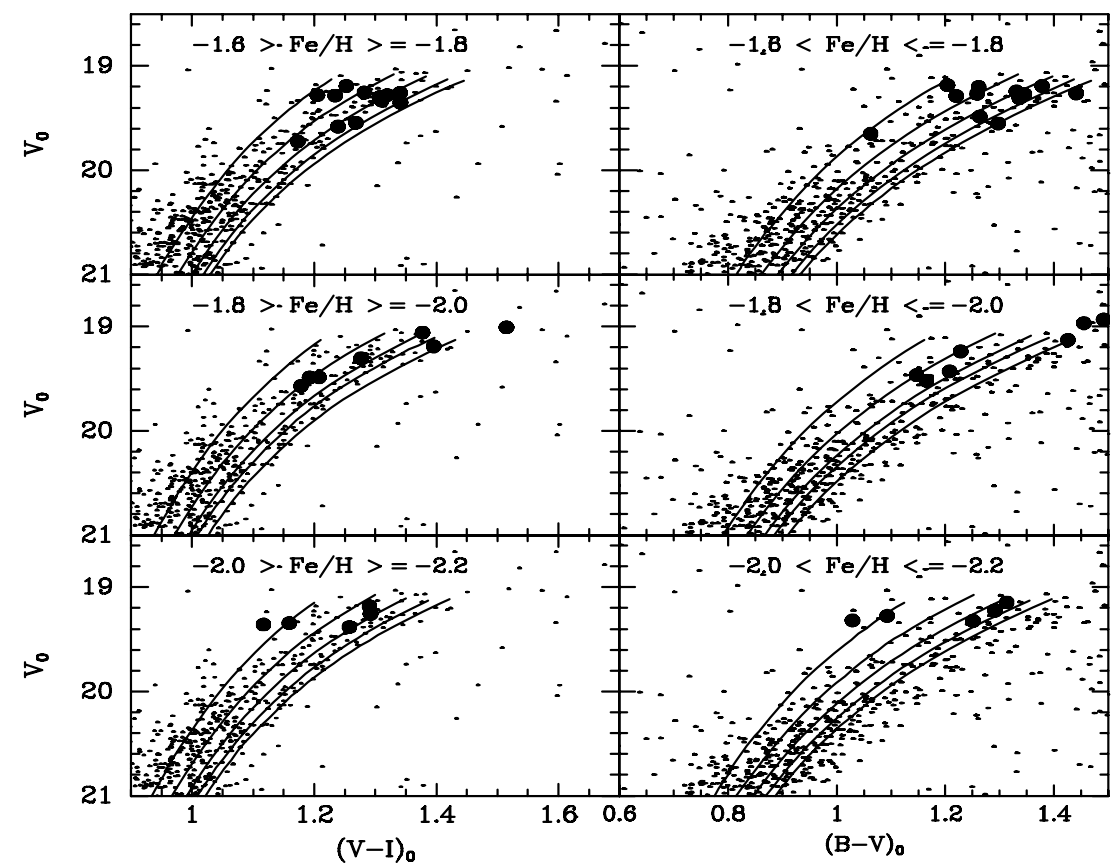

Figure 9. Calculation of approximate Leo II RGB star ages. The panels are broken down by metallicity and passband. The isochrones correspond, from bluest to reddest, to the age of 3,6,9,12, and 15 Gyr. Note the two outlying stars in the bottom panels which are likely AGB stars. These are different stars from the two outliers highlighted in Figures 3-8, which have been excluded a priori.

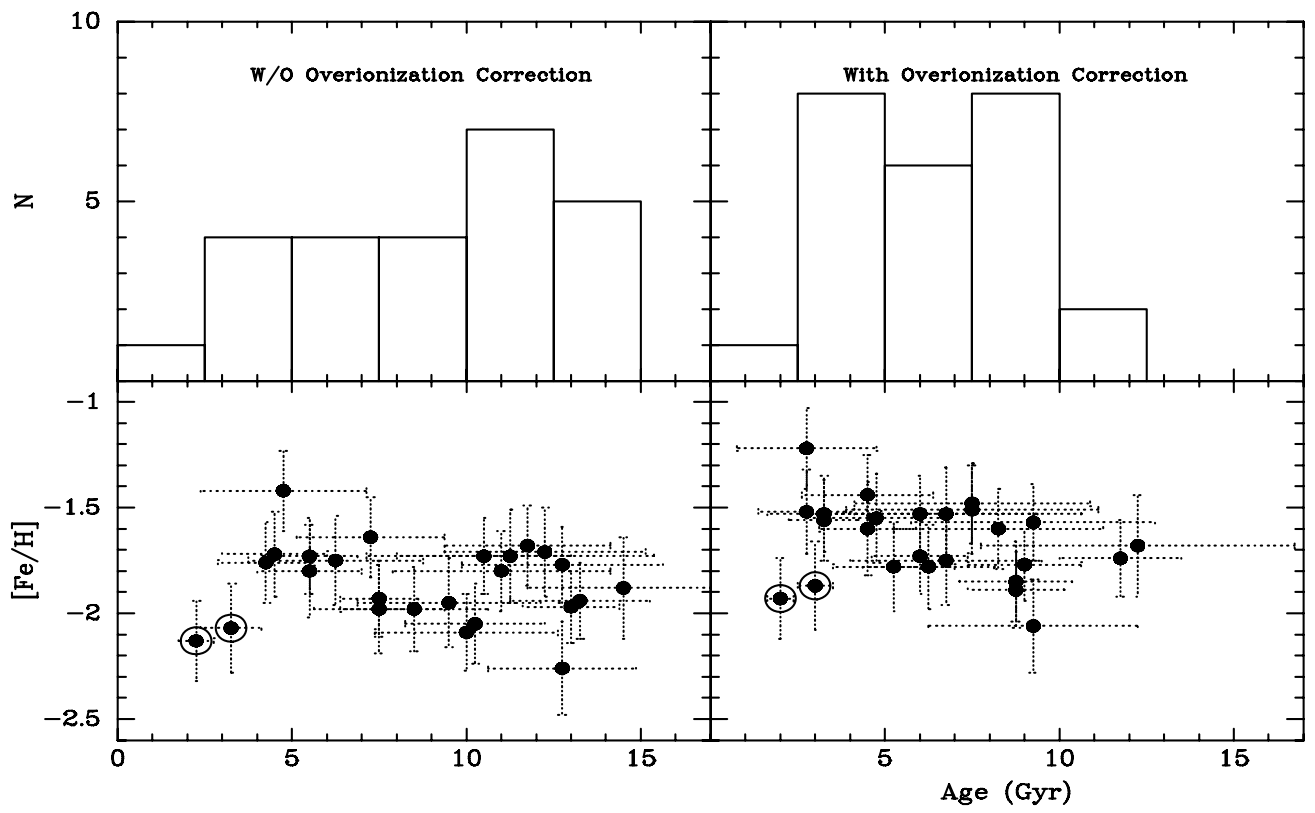

Figure 10. Age distribution and AMR for the RGB stars of Leo II. The top panels show a histogram of the inferred ages, while the lower panels show the AMR. The left panels shows the raw distributions, while the right panels are corrected for over-ionization of the Fe I lines. The circled points are the outlier stars, which are different stars than the two outliers rejected in Figures 3-8.

younger than $15 \mathrm{Gyr}$, then Leo II must be at a distance of at least $(m-M)=21.7$. Longer distances result in younger absolute ages but have little effect on the inferred AMR.

3. The over-ionization correction mentioned in Section 2 could alter the AMR by shifting the sample to a more metal-rich distribution. We applied a 0.2 dex correction (right panels of Figure 10) and find that the AMR tightens somewhat, producing a sharper peak in the age distribution at around 6-7 Gyr. However, a flat AMR would still be within the uncertainties $\left(\chi^{2}=0.9\right)$, even if the two outlier stars are removed.
4. Our S/N-limited sample is unable to probe the fainter more metal-rich stars in Leo II. Improved spectra of these stars would extend the AMR over a greater range of metallicity, providing better constraint of the enrichment and allowing a comparison to toy models of enrichment. In particular, it would determine if the apparent rise in abundance for the younger stars in Figure 10 is merely statistical scatter or is the indicator of a larger trend.

Even with these caveats, the age-metallicity distribution of Leo II stars, while giving tantalizing hints of enrichment, remain statistically consistent with no enrichment. A flat enrichment 
would be unexpected for Leo II, since we $d o$ find that the more metal-rich stars have lower $\alpha$-element abundances, suggesting gradual enrichment by SNIa over the course of several Gyr.

In the end, while this exercise demonstrates that precise photometry and spectroscopy can be used to probe the AMR of objects out to $200 \mathrm{kpc}$, the enrichment of Leo II may be too subtle to be detected in our data. Spectroscopic examination of the more numerous and age-sensitive turnoff stars may be the only way to tease out the AMR of this distant and enigmatic galaxy.

\section{DISCUSSION AND CONCLUSIONS}

Our analysis of the weaker spectral features in the BSS07 spectra provides critical information about not only the Leo II dSph galaxy but other spectroscopic studies as well.

1. We find the Fe-line metallicities disagree with CaT-based metallicities, although it is likely this is a result of the over-ionization of Fe at low metallicity. We are unable to analyze the fainter more metal-rich stars to confirm the high-metallicity tail identified by BSS07.

2. We confirm that Leo II, like the other low-luminosity dSph galaxies, has globular cluster-like $\alpha$-abundances in its most metal-poor stars but shows a declining $\alpha$-abundance at higher metallicities, likely due to the increasing influence of SNIa.

3. Leo II's AMR gives tantalizing hints of slow enrichment. However, a flat AMR with no enrichment cannot be ruled out with the present data, given the inherent uncertainties in analyzing only the brightest stars in the galaxy. Nevertheless, this demonstrates that precise spectroscopic and photometric data can be used to get a general picture of the enrichment history of distant $\mathrm{dSph}$ galaxies.

Leo II's particular influence on models of dwarf galaxy evolution lies in its difference from the other dSph galaxies. As noted in Section 1, Leo II appears to have had little interaction with the Milky Way. This would indicate that the $\alpha$-abundance patterns found in the $\mathrm{dSph}$ galaxies are a universal feature of dwarf galaxies and have no relation to any dynamical interaction with a larger parent galaxy. It also strengthens the contention that while objects like the present retinue of dSphs have contributed somewhat to the construction of the Milky Way-given the similarities between their most metal-poor stars and some chemically peculiar stars within the halo - the stark chemical dissimilarities between the bulk of the dSph and field halo stars indicates that this contribution has been small.

Further investigation is needed into the zero-point issues intrinsic in our analysis so that we may more confidently translate measured Fe I abundances into intrinsic Fe II abundances. This could be accomplished by looking at existing high $\mathrm{S} / \mathrm{N}$ CaT spectra of globular clusters, especially those known to be deficient in $\alpha$-elements or those known or suspected to have been stripped from larger $\mathrm{dSph}$ galaxies. Once the zero-point issues are understood, the technique we have unveiled in this paper can be applied to the large $\mathrm{CaT}$ spectral data sets in the literature to derive $\alpha$-abundance measures for thousands of dSph and field halo stars. Better and easier chemical fingerprinting of distant RGB stars has the potential to finally unravel the hierarchical history of the Milky Way.

Moreover, this technique expands the range of current and future investigations into $\mathrm{dSph}$ chemistry. It shows that current telescopes can probe dSph chemistry out to at least 200 $\mathrm{kpc}$ and indicates that next-generation telescopes will open up investigation into even more isolated dwarfs such as the Phoenix dIrr/dSph (Tobolewski et al. 2008, in preparation). This will expand the study of galactic chemical evolution into an even more diverse array of galactic environments. At this time, however, the existing models of dwarf galaxy chemical evolution appear to be valid out to at least $200 \mathrm{kpc}$ from the Galaxy.

This project was completed during the McDonald Observatory REU and was supported under NSF AST-0649128. M.H.S. and M.D.S. were supported by NSF grant AST-0306884.

\section{REFERENCES}

Aaronson, M., Olszewski, E. W., \& Hodge, P. W. 1983, ApJ, 267, 271

Asplund, M., \& García Pérez, A. E. 2001, A\&A, 372, 601

Azzopardi, M., Lequeux, J., \& Westerlund, B. E. 1985, A\&A, 144, 388

Battaglia, G., Irwin, M., Tolstoy, E., Hill, V., Helmi, A., Letarte, B., \& Jablonka, P. 2008, MNRAS, 383, 183

Bellazzini, M., Correnti, M., Ferraro, F. R., Monaco, L., \& Montegriffo, P. 2006, A\&A, 446,

Bellazzini, M., Gennari, N., \& Ferraro, F. R. 2005, MNRAS, 360, 185

Bosler, T. L., Smecker-Hane, T. A., \& Stetson, P. B. 2007, MNRAS, 378, 318 (BSS07)

Carretta, E., \& Gratton, R. G. 1997, A\&AS, 121, 95

Chou, M.-Y., et al. 2007, ApJ, 670, 346

Cohen, J. G., \& Meléndez, J. 2005, AJ, 129, 303

Coleman, M., Jordi, K., Rix, H.-W., Grebel, E. K., \& Koch, A. 2007, AJ, 134, 1938

Demers, S., \& Harris, W. E. 1983, AJ, 88, 329

Demers, S., \& Irwin, M. J. 1993, MNRAS, 261, 657

Dolphin, A. E., Weisz, D. R., Skillman, E. D., \& Holtzman, J. A. 2005, arXiv: astro-ph/0506430

Dotter, A., Chaboyer, B., Jevremovic, D., Baron, E., Ferguson, J., Sarajedini, A., \& Anderson, J., 2007, AJ, 134, 376

Fulbright, J. P., McWilliam, A., \& Rich, R. M. 2006, ApJ, 636, 821

Fulbright, J. P., McWilliam, A., \& Rich, R. M. 2007, ApJ, 661, 1152

Gratton, R. G., \& Ortolani, S. 1989, A\&A, 211, 41

Gullieuszik, M., Held, E. V., Rizzi, L., Girardi, K., Marigo, P., \& Momany, Y. 2008, MNRAS, 388, 1185

Helmi, A., et al. 2006, ApJ, 651, L121

Hilker, M., Kayser, A., Richtler, T., \& Willemsen, P. 2004, A\&A, 422, L9

Hinkle, K., Wallace, L., Valenti, J., \& Harmer, D. 2000, Visible and Near Infrared Atlas of the Arcturus Spectrum 3727-9300 A (San Francisco, CA: ASP)

Hodge, P. W. 1982, AJ, 87, 1668

Ivans, I. I., Kraft, R. P., Sneden, C., Smith, G. H., Rich, R. M., \& Shetrone, M. 2001, AJ, 122, 1438

Koch, A., Grebel, E. K., Kleyna, J. T., Wilkinson, M. I., Harbeck, D. R., Gilmore, G. F., Wyse, R. F. G., \& Evans, N. W. 2007a, AJ, 133, 270

Koch, A., Wilkinson, M. I., Kleyna, J. T., Gilmore, G. F., Grebel, E. K., Mackey, A. D., Evans, N. W., \& Wyse, R. F. G. 2007b, ApJ, 657, 241

Koch, A., \& McWilliam, A. 2008, AJ, 135, 1551

Kraft, R. P., \& Ivans, I. I. 2003, PASP, 115, 143

Lee, J.-W., Carney, B. W., \& Habgood, M. J. 2005, AJ, 129, 251

Lee, M. G. 1995, AJ, 110, 1155

Lee, Y.-W., Joo, J.-M., Sohn, Y.-J., Rey, S.-C., Lee, H.-C., \& Walker, A. R. 1999, Nature, 402, 55

Letarte, B., Hill, V., Jablonka, P., Tolstoy, E., François, P., \& Meylan, G. 2006, A\&A, 453, 547

Majewski, S. R., Ostheimer, J. C., Kunkel, W. E., \& Patterson, R. J. 2000, AJ, 120, 2550

Majewski, S. R., Skrutski, M. F., Weinberg, M. D., \& Ostheimer, J. C. 2003, ApJ, 599, 1082

Marigo, P., Girardi, L., Bressan, A., Groenewegen, M. A. T., Silva, L., \& Granato, G. L. 2008, A\&A, 482, 883

Marin-Franch, A., et al. 2008, ApJ, submitted

Mateo, M. L. 1998, ARA\&A, 36, 435

McWilliam, A. 1990, ApJS, 74, 1075

Mighell, K. J., \& Rich, M. R. 1996, AJ, 111, 777

Milone, A. P., et al. 2008, ApJ, 673, 241

Oke, J. B., et al. 1995, PASP, 107, 375

Palma, C., Majewski, S. R., Siegel, M. H., Patterson, R. J., Ostheimer, J. C., \& Link, R. 2003, AJ, 125, 1352

Piotto, G., et al. 2007, ApJ, 661, L53 
Pritzl, B. J., Venn, K. A., \& Irwin, M. 2005, AJ, 130, 2140

Ramírez, S. V., \& Cohen, J. G. 2002, AJ, 123, 3277

Ramírez, I., \& Meléndez, J. 2005, ApJ, 626, 465

Shetrone, M. D., Bolte, M., \& Stetson, P. B. 1998, AJ, 115, 1888

Shetrone, M. D., Côté, P., \& Sargent, W. L. W. 2001, ApJ, 548, 592

Shetrone, M. D., Venn, K. A., Tolstoy, E., Primas, F., Hill, V., \& Kaufer, A. 2003, AJ, 125, 684

Siegel, M. H., \& Majewski, S. R. 2000, AJ, 120, 284

Siegel, M. H., Majewski, S. R., Sohn, S. T., Shetrone, M. D., Munoz, R. R., \& Patterson, R. J. 2008, ApJ, submitted [S08]

Siegel, M. H., et al. 2007, ApJ, 667, L57

Simon, J. D., \& Geha, M. 2007, ApJ, 670, 313

Smith, G. H., Siegel, M. H., Shetrone, M. D., \& Winnick, R. 2006, PASP, 118, 1361

Sneden, C. 1973, ApJ, 184, 839

Sneden, C., Kraft, R. P., Guhathakurta, P., Peterson, R. C., \& Fulbright, J. P. 2004, AJ, 127, 2162
Sneden, C., Kraft, R. P., Langer, G. E., Prosser, C. F., \& Shetrone, M. D. 1994, AJ, 107, 1773

Sohn, S. T., et al. 2007, ApJ, 663, 960

Sollima, A., Pancino, E., Ferraro, F. R., Bellazzini, M., Straniero, O., \& Pasquini, L. 2005, ApJ, 634, 332

Suntzeff, N. B., Aaronson, M., Olszewski, E. W., \& Cook, K. H. 1986, AJ, 91, 1091

Swope, H. H. 1967, PASP, 79, 439

Swope, H. H. 1968, AJ, 73, S204

Thévenin, F., \& Idiart, T. P. 1999, ApJ, 521, 753

Tolstoy, E., Venn, K. A., Shetrone, M., Primas, F., Hill, V., Kaufer, A., \& Szeifert, T. 2003, AJ, 125, 707

Venn, K. A., Irwin, M., Shetrone, M. D., Tout, C. A., Hill, V., \& Tolstoy, E. 2004, AJ, 128, 1177

Vogt, S. S., Mateo, M., Olszewski, E. W., \& Keane, M. J. 1995, AJ, 109, 151

Walker, M. G., Mateo, M., Olszewski, E. W., Gnedin, O. Y., Wang, X., Sen, B., \& Woodroofe, M. 2007, ApJ, 667, L53 